\title{
Towards Structural Analysis of Polymeric Contaminants in Electrodeposited $\mathrm{Cu}$ films
}

Pavel Moreno-García, ${ }^{\mathrm{a}, 1}$ Valentine Grimaudo, ${ }^{\mathrm{a}, 1}$ Andreas Riedo, ${ }^{\mathrm{b}}$ Marek Tulej, ${ }^{\mathrm{b}}$ Maike B. Neuland, ${ }^{\mathrm{b}}$ Peter Wurz, ${ }^{\mathrm{b}}$ and Peter Broekmann ${ }^{\mathrm{a} *}$

aDepartment of Chemistry and Biochemistry, Interfacial Electrochemistry Group, University of Bern, Freiestrasse 3, 3012 Bern, Switzerland

${ }^{b}$ Physics Institute, Space Research and Planetary Sciences, University of Bern, Sidlerstrasse 5, 3012 Bern, Switzerland

\footnotetext{
*Corresponding author at: Department of Chemistry and Biochemistry, Interfacial Electrochemistry Group, University of Bern, Freiestrasse 3, 3012 Bern, Switzerland. E-mail address: peter.broekmann@dcb.unibe.ch

${ }^{1}$ These authors contributed equally to this paper
}

\begin{abstract}
:
The incorporation of plating additives into $\mathrm{Cu}$ films was studied by means of complementary Laser Ablation/Ionization Mass Spectrometry (LIMS) and Secondary Ion Mass Spectrometry (SIMS) depth profiling techniques combined with Focused Ion Beam (FIB) analysis.

$\mathrm{Cu}$ test samples were electrodeposited in the presence of a new prototypical two-component additive package for advanced Damascene applications consisting of a hybrid-type suppressor additive (Imep: polymerizate of epichlorohydrin and imidazole) and its essential co-additive (SPS: bis-(sodium-sulfopropyl)-diłulfide). The tunable non-linear interplay between the Imep and the SPS during oscillatory $\mathrm{Cu}$ electrodeposition is used to fabricate
\end{abstract}


samples bearing spatially confined layers with sequential high and marginal additive embedment. These test samples constitute an excellent platform to conduct true quantitative chemical depth profiling analysis of electrodeposited $\mathrm{Cu}$ films. In particular LIMS depth profiling measurements with an unmatched nano-meter depth-resolution demonstrate a preferential inclusion and accumulation of contaminants at grain boundaries inside the $\mathrm{Cu}$ deposit whereas the $\mathrm{Cu}$ grains remain largely contamination-free. A novel LIMS desorption approach is presented which allows for a molecular structure analysis of the polymeric additive ensembles preferentially embedded at grain boundaries of the $\mathrm{Cu}$ deposit. Our LIMS analysis confirms a recently discussed mechanism on the action of these hybrid additives which relies on their interaction with thiolate-stabilized $\mathrm{Cu}(\mathrm{I})$ intermediates.

Keywords: Electroplating, Damascene process, additives, chemical depth profiling, laser ablation/ionization mass spectrometry (LIMS).

\section{Introduction}

Additive-assisted copper electroplating is a key step in the manufacturing of cutting-edge microprocessors [1-4]. To achieve super-conformal bottom-up fill of vias and trenches, the classical Damascene approach employs a three-component package, usually consisting of a so-called type-I suppressor polymer (e.g. polyalkylene glycols, PAGs) [1, 5-11], its specific anti-suppressor (bis-(sodium-sulfopropyl)-disulfide, SPS) and a leveler additive (typeII suppressor [5]) dissolved in the $\mathrm{Cu}$ (II)-rich plating bath. Particular additive mass transport effects and adsorption kinetics [12-14] in conjunction with geometric shape-evolution effects need to be operative during the feature fill [15-19] thus generating a non-uniform surface coverage of PAG suppressors and SPS anti-suppressors. In this way, the suppressing effect of the PAG chemistry is effective predominantly at the upper feature side walls and the 
topmost wafer surface whereas the SPS accelerated copper deposition occurs preferentially at the feature bottom, thus leading to the desired differential electrodeposition. The third constituent of the electroplating bath, the so-called leveler (type-II suppressor) is meant to hinder undesired sustained copper growth (overplating, momentum plating) at the final stage of the deposition process over filled features $[20,21]$. The leveler action prevents the formation of $\mathrm{Cu}$ bumps and their coalescence over dense fields of filled trenches or vias (mounding) which is highly disadvantageous in the subsequent CMP (chemical mechanical polishing) process step [21]. Its selective and time-delayed suppressing action at the wafer surface and its immunity towards SPS deactivation are crucial requirements for the leveler action. Classical leveler approaches rely, however, on mass transport limitations in combination with a consumptive additive inclusion into the deposit during growth [22-25]. A serious drawback of such common levelers (e.g., Janus B. Green (JBG) and derivatives thereof) [26-28] is their high and non-uniform embedment into the copper deposit which significantly lowers the post-deposition self-annealing rate, i.e., recrystallization [26, 27]. The particular contamination embedment crucially depends on the local concentration of the typeII suppressor/leveler additives upon feature fill evolution.

To overcome this undesired side effect of the action of levelers, current approaches employ additives that become active more selectively over Damascene features right after the successful bottom-up fill. In this context, such more advanced approaches of leveling are not predominantly based on the classical transportation and inclusion concept but rely on sophisticated synergistic and antagonistic interactions with SPS as recently discussed [2931]. Prototypical additives exhibiting this bifunctionality are polymerizates of epichlorohydrin and imidazole (denoted Imep hereinafter, see Fig. 1).

The suppressing mode of action relies on their interaction with $\mathrm{Cu}(\mathrm{I})$-MPS complexes (see Fig. 1) which appear as side products of the SPS reaction cycle in the course of the dissociative adsorption of the SPS at the $\mathrm{Cu}$ surface during $\mathrm{Cu}$ electrodeposition. 


\section{Insert Fig. 1 (single column)}

Recent investigations demonstrated that both successful superfill and leveling can be achieved in Damascene electroplating processes even in the absence of PEG suppressors when such hybrid suppressor additives are combined with SPS [32]. Moreover, postdeposition recrystallization studies at room temperature showed large extended recrystallized domains with grain sizes beyond the $\mu \mathrm{m}$ range suggesting poor incorporation of additives when this two-additive package is employed [31]. In this contribution we demonstrate further advantages of this chemically simplified plating bath for Damascene applications by quantifying the significantly reduced inclusion of contaminants in the final copper deposits. For that purpose we conducted complementary Laser Ionization/Ablation Mass Spectrometry (LIMS) [33-38], Secondary Ion Mass Spectrometry (SIMS) and Focused Ion Beam (FIB) investigations on Imep-assisted copper electrodeposits and proved its marginal embedment into the copper matrix. Preliminary laser desorption studies performed inside layers of high additive concentration support the validity of our proposed reaction mechanism (Fig. 1).

\section{Experimental methods}

2.1 Cu electrodeposition. Chronopotentiometric measurements were carried out in a three-electrode electrochemical cell connected to an Autolab potentiostat/galvanostat (PGSTAT 128). A Ag/ $\mathrm{AgCl}_{3 \mathrm{M}}$ electrode (double junction design, Metrohm) and a Pt wire served as reference and counter electrode, respectively. The counter electrode was placed inside a glass compartment separated from the main electrochemical cell by a glass frit.

A $15.4 \mu$ m-thick $\mathrm{Cu}$ film was electrodeposited under galvanostatic conditions on a $\mathrm{Cu}$ seeded Si wafer coupon at a nominal current density $J=-6 \mathrm{~mA} \mathrm{~cm}$. The blanket wafer coupons (Hionix, provided by BASF SE) were composed of a $\mathrm{Si}(100)$-type substrate covered by $500 \mathrm{~nm}$-thick $\mathrm{TOx} / \mathrm{SiO}_{2}$ dielectric layer, a $25 \mathrm{~nm}$-thick $\mathrm{Ta} / \mathrm{TaN}$ barrier and an outermost $100 \mathrm{~nm}$-thick $\mathrm{Cu}$ seed layer. The plating bath was prepared by dissolution of $40 \mathrm{~g} / \mathrm{L}$ 
$\mathrm{Cu}^{2+}\left(\mathrm{CuSO}_{4} * 5 \mathrm{H}_{2} \mathrm{O}\right.$, Sigma-Aldrich, Steinheim, Germany), $10 \mathrm{~g} / \mathrm{L} \mathrm{H}_{2} \mathrm{SO}_{4}$ (VLSI selectipur 96\%, BASF, Ludwigshafen, Germany) and $50 \mathrm{ppm} \mathrm{Cl}$ ( $\mathrm{HCl} \mathrm{25 \%}$ for analysis, Merck, Darmstadt, Germany) in Milli-Q water (nominal resistivity of $18.2 \mathrm{M} \Omega \mathrm{cm}, \mathrm{TOC} \leq 3 \mathrm{ppb}$ ). The concentrations of SPS (Raschig, Ludwigshafen, Germany) and Imep (BASF, Ludwigshafen, Germany) in the electrolyte were 25 and 100 ppm, respectively. All chemicals were used as received without any further purification.

2.2 SIMS depth profiling. Secondary Ion Mass Spectrometry (SIMS) depth profiling studies were carried out by a Cameca ims-4f apparatus (IFOS, Kaiserslautern, Germany). $\mathrm{Cs}^{+}$cations with a kinetic energy of $E_{\text {kin }}=14.5 \mathrm{keV}$ and $5.5 \mathrm{keV}$ served as primary ions for negative and positive $\mathrm{MCs}^{+}$SIMS modes, respectively. Negative and $\mathrm{MCs}^{+}$modes differ in the polarity of detected secondary ions ( $\mathrm{M}$ being the element to be analyzed). The sputter current density and the analyzed area on the sample surface were $0.32 \mathrm{~mA} \mathrm{~cm}{ }^{-2}$ and $60 \mu \mathrm{m}$ in diameter, respectively. A typical crater formed after erosion by the SIMS technique is shown in Fig. 2a.

\section{Insert Fig. 2 (single column)}

2.3 LIMS depth profiling. Mass spectrometric studies were conducted by using a home-built miniature $(160 \mathrm{~mm} \times \varnothing 60 \mathrm{~mm})$ reflectron-type time-of-flight (TOF) laser mass spectrometer (LMS) [33-37]. An ultrashort pulsed laser system (pulse width $\tau \sim 190 \mathrm{fs,}$ wavelength $\lambda=775 \mathrm{~nm}$, laser pulse repetition rate $\leq 1 \mathrm{kHz}$ ) is used as an ablation ionization source [35]. The laser system is located outside the vacuum chamber, and an optical system is used for beam shaping and delivery to the mass spectrometer located within the vacuum chamber. Laser pulse energy, laser pulse repetition rate, and number of laser pulses are fully controlled by the interface of the laser system. A doublet lens on top of the mass analyzer is used to focus the laser beam throfigh the system on the sample surface (laser ablation crater with a diameter of about $18.7 \mu \mathrm{m})$. The laser focus, and thus the sample 
surface, is positioned close to the entrance of the ion-optical system, about $1 \mathrm{~mm}$ away. A high-resolution $\mathrm{x}-\mathrm{y}-\mathrm{z}$ micro translation stage with a position accuracy of about $2 \mu \mathrm{m}$ is used for the positioning of samples relative to the mass spectrometer. Only positively charged ions, generated by the interaction of the laser pulse with the sample surface, can enter the mass analyzer. Entering the mass analyzer the ions are accelerated, focused, and confined in the instrument by means of an ion optical system. Positive ions pass first the field free region before they are reflected by the ion mirror towards the detector system. The ions arrive at the detector system sequentially in time according to their mass to charge $(\mathrm{m} / \mathrm{z})$ ratio (TOF principle), where they produce electrical signals. Two high speed ADC systems (each with two channels, 8 bit vertical resolution, up to $4 \mathrm{GS} / \mathrm{s}$ ) are used for the acquisition of the electric signal, the TOF spectrum (length of $20 \mu \mathrm{s}$ ). Once the measurement campaign is accomplished, the TOF spectra are converted to mass spectra $(\mathrm{m} / \mathrm{z})$ by using the relation $(m / z)(t)=a\left(t-t_{0}\right)^{2}$, where $a$ and $t_{0}$ are calibration constants [33]. Typically, an entire measurement campaign consists of up to several thousands of single laser shots mass spectra. The current measurement setup allows acquiring and saving single laser shot mass spectra on the host computer and its real time visualization on a screen. Custom-made analysis software, written in MATLAB, is used for subsequent data analysis.

To obtain statistically significant data, the LIMS depth-profile analysis was carried out on a $1.96 \mathrm{~mm}^{2}$ area comprising 49 scrutinized locations each separated by $200 \mu \mathrm{m}$ from the closest neighbors. A laser irradiance of $3.29 \mathrm{TW} \mathrm{cm}^{-2}(3.40 \mu \mathrm{J}$ per pulse) was applied to ablate the copper film stepwise until the $\mathrm{Si}$ signal from the underlying $\mathrm{SiO}_{2}$ substrate was detected. The mean ablation rate under these conditions was $9.7 \pm 1.5 \mathrm{~nm} \mathrm{shot}^{-1}$. Craters formed upon sample erosion have an average diameter of $18.7 \pm 1.1 \mu \mathrm{m}$. A typical SEM image of LIMS craters is displayed in Fig. 2b. Each measurement was conducted on a fresh and untreated sample location. The depth profile for each element was acquired by 
analyzing the temporal evolution of the signal intensity of a selected element/isotope in the TOF spectra [34]. With the knowledge of the $\mathrm{Cu}$ layer thickness and number of applied laser shots the mean ablation rate per single laser shot was derived.

2.4 LIMS desorption studies. To directly prove that the impurity layers periodically embedded into the $\mathrm{Cu}$ film are in fact composed by the Imep-Cu(I)-MPS suppressor complexes (see Fig. 1) we additionally performed so-called laser desorption studies at these layers which allowed us to identify the respective monomeric building block of the polymeric suppressor complex $(m / z=342)$ as a fragment resulting from the laser desorption process. These experiments consisted of two subsequent campaigns differing in their laser irradiances. In a first stage, harsh ablation conditions $\left(\sim 2.5 \mathrm{TWcm}^{-2}\right)$ were applied to the sample until an impurity layer was reached upon erosion. This was manifested by an abrupt drop of the $\mathrm{Cu}$ signal and a correlated increase of the $\mathrm{C}, \mathrm{O}, \mathrm{N}$ and $\mathrm{S}$ intensities. The laser ablation of material was then stopped and the laser irradiance was decreased to lower values just above the socalled desorption threshold $\left(0.4-0.6 \mathrm{TW} \mathrm{cm}^{-2}\right)$. In this way, sample material was desorbed in a soft manner avoiding complete atomization of the polymeric suppressor ensembles thus allowing for its structural and molecular analysis.

2.5 SEM analysis. The surface morphology of the $\mathrm{Cu}$ samples after SIMS and LIMS depth profiling analysis was characterized by a Hitachi S-3000 N Scanning Electron Microscope (SEM).

\section{Results and discussion}

Recent mechanistic studies proposed an adduct of the Imep polymer with $\mathrm{H}_{2} \mathrm{O}-\mathrm{Cu}(\mathrm{I})-\mathrm{MPS}$ complexes as the active suppressor ensemble being operative at the interface during $\mathrm{Cu}$ plating in the presence of the two-component Imep/SPS additive package $[29,30]$. The $\mathrm{H}_{2} \mathrm{O}-\mathrm{Cu}(\mathrm{I})-$ MPS complex is a byproduct of the SPS which undergoes adsorptive dissociation on the $\mathrm{Cu}$ surface $[29,30]$. A combination of $\mathrm{Cu}(\mathrm{I})$ coordination coupled to inner-salt formation and 
inter-chain anion/cation pairing has been proven to be the chemical origin of the surfaceconfined precipitation of these suppressor/leveler adducts (Fig. 1). To determine to which extent and under which experimental conditions these species get incorporated into the copper film upon electrodeposition, we make use of a particular nonlinear instability of the Imep/SPS additive system that occurs under specific galvanostatic plating conditions [29, 30]. The physical origin of these temporal instabilities is a so-called $\mathrm{N}$-shaped negative differential resistance (N-NDR) $[39,40]$ that, within a relatively narrow current range, is manifested by potential oscillations during chronopotentiometric measurements.

\section{Insert Fig. 3 (single column)}

Fig. 3a displays the potential versus charge transient of a $15.4 \mu \mathrm{m}$-thick copper film electrodeposited on a blanket wafer coupon at a nominal current density of $J=-6 \mathrm{~mA} \mathrm{~cm}$. The plot shows seven regular potential oscillations that are attributed to sequential formation of the stable Imep-Cu(I)-MPS suppressor film and its partial dissolution (deactivation) at the interface $[29,30]$. The intervals at higher overpotentials comprised between sharp potential steps correspond to an active Imep-Cu(I)-MPS diffusional barrier being operative at the interface. The abrupt decrease and following abrupt increase of the deposition overpotential are indicative for the partial or complete breakdown and subsequent restoration of the suppressor ensemble, respectively. It has already been demonstrated that the suppressing ensemble incorporates into the copper matrix to a substantial extent during the sharp potential transitions whereas the $\mathrm{Cu}$ deposited under quasi-steady state deposition remains almost impurity-free [31]. As a consequence, post-deposition recrystallization of such samples extends over large domains and is typically confined by the localized contamination layers. The resulting sample structure bearing multiple layers of embedded impurities perpendicular to the growth direction makes it an ideal platform for chemical depth profiling studies. To correlate the oscillatory behavior observed in the chronopotentiometry with the final film 
morphology, we performed FIB analyses. Fig. 3b shows a typical cross-sectional FIB micrograph that reveals an almost periodic array of extended grain boundaries normal to the growth direction separating well recrystallized domains of $\mathrm{Cu}$ inside the deposited film. We assign the observed sharp deltas to spatially confined copper layers bearing high contamination levels that hinder further coalescence of neighboring copper grains. We note, however, that only six such deltas are discernible in the FIB image. The two closely spaced transitions at the initial stage of the galvanostatic experiment are merged together. To further support the assignment of these localized boundaries to the incorporation of the suppressor ensemble we conducted SIMS depth profiling studies which determined the contamination level of $\mathrm{C}, \mathrm{O}, \mathrm{N}$ and $\mathrm{S}$ along the surface normal of the sample (Fig. $3 \mathrm{c}$ ). Based on white light interferometry analysis of the SIMS craters we determined a sample depth of $14.0 \mu \mathrm{m}$, which agrees well with the nominal $\mathrm{Cu}$ film thickness calculated on the basis of the coulometric data (Fig. 3a). This slight thickness underestimation has already been observed in previous studies and might be ascribed to a certain non-uniformity in the $\mathrm{Cu}$ film across the surface of the wafer coupon [31]. Nevertheless, the SIMS depth profiles show an inphase modulation of the peak-centered $\mathrm{C}, \mathrm{O}, \mathrm{N}$ and $\mathrm{S}$ signals with progressive erosion depth that correlates with the oscillatory characteristics in the potential/charge transient curve and the layer boundaries in the FIB image. Deconvolution of the two features at the closest vicinity with the wafer coupon was, however, not possible which correlates with the absence of two distinguishable impurity layers at that sample depth in the FIB micrograph (Fig. 3a).

The observed strong attenuation of the SIMS amplitudes with increasing erosion depth is a well-known phenomenon which can usually be ascribed to a sputtered-induced surface roughening and layer intermixing upon erosion that accompanies an increase in the full width at half maximum (FWHM) of SIMS peaks. This peak broadening in the SIMS experiment contrasts with the sharpness of the potential/charge steps and the localized layer boundaries in Fig. 3a and b, respectively. Fig. 2a shows an SEM micrograph of the eroded 
area after ion bombardment with the SIMS technique which clearly proves the significant roughening of the $\mathrm{Cu}$ surface upon ion erosion.

One further drawback of the SIMS technique is the strong dependence of the signal intensities on the measuring mode, namely negative ion and $\mathrm{MCs}^{+}$modes. The impact of this undesired effect is misleading with regard to the quantification of $\mathrm{N}$ impurities, which leads to intensities that differ by up to three orders of magnitude (Fig. 3c). This is particularly disadvantageous for assessment of leveler embedment into $\mathrm{Cu}$ interconnects since $\mathrm{N}$ atoms are usually an essential component of such leveler additives (type-II suppressors [5]). In our particular case this issue becomes evident if we consider all species of the SIMS profile measured using both negative ion and $\mathrm{MCs}^{+}$modes. In Fig. $3 \mathrm{c}$ we see that the $\mathrm{N}$ signal in negative ion mode (detection of $\mathrm{N}$ via $\mathrm{CN}^{-}$anions) overwhelms the intensities of all other elements. This picture is not consistent with the suggested incorporation of the suppressing ensemble shown in Fig. 1. Instead, a significantly lower concentration of $\mathrm{N}$ in comparison to $\mathrm{C}$ and $\mathrm{O}$ is expected. However, when only $\mathrm{MCs}^{+}$mode SIMS data were considered, we obtained an improved qualitative trend with $\mathrm{N}$ being the least abundant species inside the $\mathrm{Cu}$ sample. Therefore, we suggest that the $\mathrm{MCs}^{+}$mode of the SIMS approach enables a more accurate assignment of impurity concentrations.

To overcome these inherent limitations of the SIMS technique and to determine the actual incorporation of the suppressing complex into the final copper deposit, we made use of a truly quantitative, sensitive (10 ppb element/isotope concentration in sample material, atomic fraction) LIMS depth profiling instrument that can achieve even sub-nanometer vertical resolution [33-38]. The capabilities of this technique on the analysis of copper electrodeposits prepared with the Imep-SPS additive package were recently proven for multilayered samples that were also prepared by an oscillatory $\mathrm{Cu}$ electrodeposition [41]. In that former study we, however, restricted ourselves to the determination of impurities inside the highly contaminated sections of the periodic structure. We resolved a detailed and 
quantitative contamination depth profile with unprecedented high vertical resolution that was not severely affected by the ablation process as in the case of the SIMS experiments (see comparison of SIMS and LIMS craters in Fig. 2). Herein we extend such quantitative analysis to the zones in the $\mathrm{Cu}$ film deposited under quasi-steady state conditions.

\section{Insert Fig. 4 (single column)}

Fig. 4 shows, as a representative example, the $\mathrm{Cu}$ and $\mathrm{O}$ signals of a typical LIMS depth profile. The high O intensity at the beginning of the LIMS depth profile can be assigned to the native oxide layer on the surface formed in air after the $\mathrm{Cu}$ deposition. Therefore we do not consider this $\mathrm{O}$ contribution in our quantitative analysis of the LIMS data. We assign the observed six abrupt periodic anti-correlated peaks in the $\mathrm{Cu}$ and $\mathrm{O}$ depth profiles (highlighted in Fig. 4 by grey bars) to the highly localized contamination layers that correspond to the sharp deltas in the FIB micrograph of Fig. 3b. At this point it should be noted that the measured intensities of the other elements under consideration $(\mathrm{C}, \mathrm{N}, \mathrm{S}$ and $\mathrm{Cl})$ are indeed in phase with the O signal in full agreement with the SIMS depth profiling results (Fig. 3c). These depth profiles are considered for the quantitative LIMS analysis but are, for the sake of clarity, not included into Fig. 4.

Our general approach towards a more detailed quantitative analysis of the LIMS depth profiles is presented in Fig. 5 showing an enlarged section of one complete O LIMS depth profile.

\section{Insert Fig. 5 (single column)}

For the quantification of contaminants we divided the depth profiles in three sub-sections denoted in Fig. 5 as L, B and S. L refers to the regimes of highest contamination inside the $\mathrm{Cu}$ film (highlighted blue in Fig. 5) and corresponds to the sharp delta layers (L) seen in the FIB cut of Fig. 3b. B refers to the background impurity level (highlighted grey in Fig. 5) representing the regime between those deltas in the LIMS depth profile and corresponds to the 
well recrystallized $\mathrm{Cu}$ (Fig. 3b). In addition there are smaller satellite peaks superimposed on the background impurity level (highlighted red in Fig. 5) that are denoted as S. Our criterion to discriminate impurity contributions from $\mathrm{S}$ and $\mathrm{B}$ was a threshold of a minimum two-fold relative increase of the LIMS signal for all major contaminant species $(\mathrm{O}, \mathrm{C}, \mathrm{N}, \mathrm{S}$ and $\mathrm{Cl})$ going along with an according sharp decrease of the $\mathrm{Cu}$ signal.

For the impurity quantification we integrated the individual element intensities $(\mathrm{O}, \mathrm{C}, \mathrm{N}, \mathrm{S}$ and $\mathrm{Cl}$ ) separately for L, B and S regimes in the range from the sample surface (excluding the native oxide layers) down to the dielectric. The result of this analysis is presented in Tab. 1 and Fig. 6.

\section{Insert Table. 1 (single column)}

\section{Insert Fig. 6 (single column)}

The general characteristics of the depth profiles can be rationalized under the assumption that embedded impurities inside the $\mathrm{Cu}$ film are preferentially accumulated at grain boundaries. As one can expect, the total impurity concentration (in ppm) is highest for $\mathrm{L}\left(\mathrm{C}_{\mathrm{tot}}=36800 \mathrm{ppm}\right)$ where the ablated material originates from a single grain boundary, which matches with the plane of laser irradiation and the resulting layer of ablated material in the LIMS depth profiling process. The chemical composition of $\mathrm{L}$ is therefore dominated by contributions from the deltatype grain boundary itself with an insignificant portion of ablated material originating from the $\mathrm{Cu}$ bulk phase. For this reason a determination of the chemical composition of the grain boundary including true quantification becomes feasible. A crucial prerequisite for that is a mean ablation rate of the LIMS experiment that is smaller than the spatial extension of the grain boundary in direction of the depth profiling process.

This situation is just inversed for the regimes B and S in Fig. 5 where the majority of ablated 12

material originates from clean $\mathrm{Cu}$ grains with only minor contributions coming from grain boundaries. The plane of laser irradiation is not parallel to a single grain boundary as in case $\mathrm{L}$ 
but intersects locally with grain boundaries that are randomly distributed in the recrystallized domains of the $\mathrm{Cu}$ film. Much smaller than one order of magnitude are therefore the impurities associated to the satellite peak regimes $\mathrm{S}\left(\mathrm{C}_{\text {tot }}=1130 \mathrm{ppm}\right)$ in the LIMS depth profile (Fig. 5) followed by the low but still well detectable background level $\mathrm{B}\left(\mathrm{C}_{\mathrm{tot}}=158 \mathrm{ppm}\right)$. Important to note is that our analysis does not necessarily imply that the local impurity concentration is lower at those grain boundaries within the domains of recrystallized $\mathrm{Cu}$ compared to the sharp delta-type layers (L). For the domains of recrystallized $\mathrm{Cu}$, the LIMS technique provides true quantitative information on impurities located in the layer of ablation. With this information we can derive the relative chemical composition of the grain boundaries (assuming that the impurities indeed originate from grain boundaries). The total impurity concentration located at those grain boundaries is, however, not accessible. The latter would require a normalization of the experimentally determined impurity concentration to the number density of domain boundaries within the layer of ablation analyzed. This is schematically demonstrated in the representative FIB micrograph of the recrystallized $\mathrm{Cu}$ in Fig. 7.

\section{Insert Fig. 7 (single column)}

A closer inspection of Tab. 1 and Fig. 6 lets us conclude that the chemical compositions of regimes $\mathrm{B}$ and $\mathrm{S}$ (relative abundances of elements) in the depth profile are qualitatively the same. In both cases it is the oxygen which is the predominant impurity species $\left(\mathrm{S}\right.$ : $\mathrm{C}_{\mathrm{O}, \mathrm{Rel}}=$ 51.89 at $\%$; $\mathrm{B}: \mathrm{C}_{\mathrm{O}, \mathrm{Rel}}=46.4$ at $\%$ ). Origin of this high oxygen level could be the embedment of partially solvated/hydrated sulfate anions from the supporting electrolyte and the inclusion of

partially solvated sulfonic functionalities of the SPS upon growth. Also the Imep polymers contain a certain amount of oxygen in form of hydroxy functionalities (see Fig. 1). The embedment of the Imep polymer becomes manifested by the relatively high carbon content (S: $\mathrm{C}_{\mathrm{C}, \mathrm{Rel}}=27.83$ at\%; B: $\mathrm{C}_{\mathrm{C}, \mathrm{Rel}}=28.2$ at $\left.\%\right)$ and the presence of nitrogen $\left(\mathrm{S}: \mathrm{C}_{\mathrm{N}, \mathrm{Rel}}=12.56\right.$ at $\%$; $\mathrm{B}$ : $\mathrm{C}_{\mathrm{N}, \mathrm{Rel}}=8.5 \mathrm{at} \%$ ). The nitrogen can be considered as a fingerprint for the embedment of the 
Imep polymer since the leveler (type-II suppressor) is the only N-containing species present in the plating bath. The comparison of the chemical composition of regimes $\mathrm{B}$ and $\mathrm{S}$ let us conclude that the impurities can be in both cases associated to the same type of grain boundaries. Their number density is assumed to be locally higher in regime $\mathrm{S}$ compared to regime $\mathrm{B}$. The appearance of satellite peaks in the regime between two sharp delta peaks in the LIMS depth profile can therefore be rationalized in terms of marginal (local) variations of the number density of grain boundaries of the same type upon laser ablation. In addition, local variations of the grain boundary orientations with respect to the direction of the laser ablation process might also lead to variations in the element abundances as seen in the LIMS depth profile (Fig. 5). These phenomena are depicted in Fig. 7.

Compared to the grain boundaries inside the recrystallized $\mathrm{Cu}$ domains we observed a different chemical composition for the delta-type grain boundaries $(\mathrm{L})$. The carbon content $\left(\mathrm{L}: \mathrm{C}_{\mathrm{C}, \mathrm{Rel}}=\right.$ $47.0 \mathrm{at} \%)$ is substantially higher and the oxygen content correspondingly lower $\left(\mathrm{L}: \mathrm{C}_{\mathrm{C}, \mathrm{Rel}}=\right.$ 33.0 at $\%$ ) compared to the grain boundaries inside the recrystallized $\mathrm{Cu}$ domains (B and $\mathrm{S}$ regimes) whereas the $\mathrm{N}, \mathrm{S}$ and $\mathrm{Cl}$ contents are comparable to the ones in the $\mathrm{S}$ and $\mathrm{B}$ regimes.

We assume that the chemical composition of the of delta-type grain boundary is governed by the breakdown and subsequent embedment of the Imep-Cu(I)-MPS suppressor ensemble (Fig. 1). Special attention should therefore be paid to the particular $C_{\mathrm{N}, \mathrm{Rel}} / C_{\mathrm{S}, \mathrm{Rel}}$ ratio. Based on the scenario depicted in Fig. 1 one would expect a 1:1 inclusion of $\mathrm{N}$ and $\mathrm{S}$. We determine, however, a $C_{\mathrm{N}, \mathrm{Rel}} / C_{\mathrm{S}, \mathrm{Rel}}$ ratio of $\sim 1.5$, suggesting that a certain amount of Imep hydroxy sites are not coordinated with $\mathrm{Cu}(\mathrm{I})$-MPS units $[29,30]$. This points to a non-stoichiometric precipitation of the Imep-Cu(I)-MPS complex under reactive conditions. An elemental analysis of the Imep-Cu(I)-MPS complex formed on a beaker scale came to the same conclusion [30].

\section{Insert Fig. 8 (double column)}

To directly prove that the impurity layers (L) embedded inside the $\mathrm{Cu}$ deposit are in fact 
composed by Imep-Cu(I)-MPS ensembles, we carried out preliminary laser desorption studies with the LMS instrument which allows for a more detailed molecular and structural characterization of an embedded impurity layer beyond the analysis of its elemental composition. Fig. 8a shows the employed experimental approach. In a first stage we ablated the $\mathrm{Cu}$ deposit (laser irradiance $\sim 2.5 \mathrm{TW} \mathrm{cm}^{-2}$ ) until an impurity layer (L) was detected as indicated by an abrupt decrease of the $\mathrm{Cu}$ signal going along with the simultaneous rise of the $\mathrm{C}, \mathrm{O}, \mathrm{N}$ and $\mathrm{S}$ intensities (Fig. 8a). The laser irradiation was then temporarily stopped and subsequently resumed after setting the laser conditions just above the desorption threshold (0.4-0.6 $\left.\mathrm{TW} \mathrm{cm}^{-2}\right)$. While laser ablation causes the atomization of the ablated material, laser desorption allows for the registration of complete molecules and their fragments. With this approach, we were for the first time able to detect higher molecular masses in the LIMS that we ascribe to characteristic fragments of the Imep-Cu(I)-MPS complex (Fig. 8b) desorbed from one of the embedded impurity layers (L) inside the $\mathrm{Cu}$ sample. The mass spectra in Fig. $8 \mathrm{~b}$ represent single laser shot LIMS spectra showing characteristic masses of the monomeric Imep-Cu(I)-MPS unit $(m / z=342)$ and a further fragment $(\mathrm{m} / \mathrm{z}=328)$ after loss of a $\mathrm{CH}_{2}$ unit.

We notice that only a low percentage of the data acquired in desorption mode $(\sim 5 \%)$ rendered such large masses and that more intense signals were observed for $\mathrm{C}, \mathrm{O}, \mathrm{N}, \mathrm{S}$ elements and smaller molecular fragments. The reason for this might be the damage induced to the deltaimpurity layer (L) in the initial ablation step leaving behind only a small fraction of intact Imep-Cu(I)-MPS network. Nevertheless, these results constitute the first direct structural evidence supporting our proposed reaction mechanism shown in Fig. 1.

\section{Conclusions}

We studied the embedment of advanced plating additives into $\mathrm{Cu}$ test samples that have been electrodeposited under oscillatory galvanostatic conditions. By means of SIMS depth profiling 15

we could prove a preferential additive incorporation under transient conditions during the 
break-down and subsequent restoration of the active suppressor ensemble whereas clean and well recrystallized $\mathrm{Cu}$ domains were obtained under quasi-steady-state conditions where the active suppressor (leveler) film is operative. The negative SIMS mode was demonstrated to be unsuitable for estimating the actual $\mathrm{N}$ content in the $\mathrm{Cu}$ sample. More accurate determination of the $\mathrm{N}$ content can be achieved by the positive $\mathrm{MCs}^{+}$SIMS mode.

We showed that a true quantification of impurity embedment becomes feasible by using advanced LIMS depth profiling techniques. Our LIMS study demonstrated a preferential embedment of contaminants at the grain boundaries of the $\mathrm{Cu}$ film. For the first time, a true determination of the chemical composition of a single grain boundary inside a $\mathrm{Cu}$ sample was achieved. A novel laser desorption mode of the LIMS technique allows for a structural analysis of the embedded suppressor ensembles beyond pure compositional (elemental) analysis of the bulk and gain boundaries of the $\mathrm{Cu}$ samples.

\section{Acknowledgements}

This work was supported by the Swiss National Science Foundation.

\section{References}

[1] P.C. Andricacos, C. Uzoh, J.O. Dukovic, J. Horkans, H. Deligianni, Damascene copper electroplating for chip interconnections, IBM J. Res. Dev., 42 (1998) 567-574.

[2] R. Akolkar, C.-C. Cheng, R. Chebiam, A. Fajardo, V. Dubin, Advanced Electrochemical Processes for Sub-50 nm On-chip Metallization, ECS Transactions, 2 (2007) 13-18.

[3] V.M. Dubin, R. Akolkar, C.C. Cheng, R. Chebiam, A. Fajardo, F. Gstrein, Electrochemical materials and processes in Si integrated circuit technology, Electrochim. Acta, 52 (2007) 28912897.

[4] X. Gagnard, T. Mourier, Through silicon via: From the CMOS imager sensor wafer level package to the 3D integration, Microelectron. Eng., 87 (2010) 470-476.

[5] P. Broekmann, A. Fluegel, C. Emnet, M. Arnold, C. Roeger-Goepfert, A. Wagner, N.T.M. Hai, D. Mayer, Classification of suppressor additives based on synergistic and antagonistic ensemble effects, Electrochim. Acta, 56 (2011) 4724-4734.

[6] J.J. Kelly, A.C. West, Copper Deposition in the Presence of Polyethylene Glycol: I. Quartz Crystal Microbalance Study, J. Electrochem. Soc., 145 (1998) 3472-3476.

[7] J.W. Gallaway, M.J. Willey, A.C. West, Copper Filling of $100 \mathrm{~nm}$ Trenches Using PEG, PPG, and a Triblock Copolymer as Plating Suppressors, J. Electrochem. Soc., 156 (2009) D287-D295.

[8] J. Mendez, R. Akolkar, U. Landau, Polyether Suppressors Enabling Copper Metallization of 
High Aspect Ratio Interconnects, J. Electrochem. Soc., 156 (2009) D474-D479.

[9] T.P. Moffat, D. Wheeler, D. Josell Electrodeposition of Copper in the SPS-PEG-Cl Additive System: I. Kinetic Measurements: Influence of SPS, J. Electrochem. Soc., 151 (2004) C262C271.

[10] J.P. Healy, D. Pletcher, M. Goodenough, The chemistry of the additives in an acid copper electroplating bath: Part I. Polyethylene glycol and chloride ion, J. Electroanal. Chem., 338 (1992) 155-165.

[11] M.R.H. Hill, G.T. Rogers, Polyethylene glycol in copper electrodeposition onto a rotating disk electrode, Journal of Electroanalytical Chemistry and Interfacial Electrochemistry, 86 (1978) 179-188.

[12] R. Akolkar, U. Landau, A Time-Dependent Transport-Kinetics Model for Additive Interactions in Copper Interconnect Metallization, J. Electrochem. Soc., 151 (2004) C702C711.

[13] R. Akolkar, U. Landau, Mechanistic Analysis of the "Bottom-Up" Fill in Copper Interconnect Metallization, J. Electrochem. Soc., 156 (2009) D351-D359.

[14] J. Adolf, U. Landau, Predictive Analytical Fill Model of Interconnect Metallization Providing Optimal Additives Concentrations, J. Electrochem. Soc., 158 (2011) D469-D476. [15] T.P. Moffat, D. Wheeler, M.D. Edelstein, D. Josell, Superconformal film growth: Mechanism and quantification, IBM J. Res. Dev., 49 (2005) 19-36.

[16] A.C. West, S. Mayer, J. Reid, A Superfilling Model that Predicts Bump Formation, Electrochem. Solid-State Lett., 4 (2001) C50-C53.

[17] A.C. West, Theory of Filling of High-Aspect Ratio Trenches and Vias in Presence of Additives, J. Electrochem. Soc., 147 (2000) 227-232.

[18] T.P. Moffat, D. Wheeler, S.-K. Kim, D. Josell, Curvature Enhanced Adsorbate Coverage Model for Electrodeposition, J. Electrochem. Soc., 153 (2006) C127-C132.

[19] T.P. Moffat, D. Wheeler, S.K. Kim, D. Josell, Curvature enhanced adsorbate coverage mechanism for bottom-up superfilling and bump control in damascene processing, Electrochim. Acta, 53 (2007) 145-154.

[20] W.-P. Dow, C.-W. Liu, Evaluating the Filling Performance of a Copper Plating Formula Using a Simple Galvanostat Method, J. Ectrochem. Soc., 153 (2006) C190-C194.

[21] J.D. Reid, J. Zhou, Impact of Leveler Molecular Weight and Concentration on Damascene Copper Electroplating, ECS Trans., 2 (2007) 77-92.

[22] D. Roha, U. Landau, Mass Transport of Leveling Agents in Plating: Steady-State Model for Blocking Additives, J. Electrochem Soc., 137 (1990) 824-834.

[23] C. Madore, M. Matlosz, D. Landolt, Blocking Inhibitors in Cathodic Leveling: I.

Theoretical Analysis, J. Electrochem Soc., 143 (1996) 3927-3936.

[24] C. Madore, D. Landolt, Blocking Inhibitors in Cathodic Leveling: II. Experimental Investigation, J. Electrochem Soc., 143 (1996) 3936-3943.

[25] J.J. Kelly, C. Tian, A.C. West, Leveling and Microstructural Effects of Additives for Copper Electrodeposition, J. Electrochem Soc., 146 (1999) 2540-2545.

[26] O. Lühn, A. Radisic, P.M. Vereecken, C. Van Hoof, W. Ruythooren, J.-P. Celis, Changing Superfilling Mode for Copper Electrodeposition in Blind Holes from Differential Inhibition to Differential Acceleration, Electrochem. Solid-State Lett., 12 (2009) D39-D41.

[27] O. Lühn, C. Van Hoof, W. Ruythooren, J.P. Celis, Filling of microvia with an aspect ratio of 5 by copper electrodeposition, Electrochim. Acta, 54 (2009) 2504-2508.

[28] W.-P. Dow, C.-C. Li, Y.-C. Su, S.-P. Shen, C.-C. Huang, C. Lee, B. Hsu, S. Hsu, Microvia filling by copper electroplating using diazine black as a leveler, Electrochim. Acta, 54 (2009) 5894-5901.

[29] N.T.M. Hai, K.W. Krämer, A. Fluegel, M. Arnold, D. Mayer, P. Broekmann, Beyond interfacial anion/cation pairing: The role of $\mathrm{Cu}(\mathrm{I})$ coordination chemistry in additive-controlled copper plating, Electrochim. Acta, 83 (2012) 367-375. 
[30] N.T.M. Hai, J. Odermatt, V. Grimaudo, K.W. Krämer, A. Fluegel, M. Arnold, D. Mayer, P. Broekmann, Potential Oscillations in Galvanostatic Cu Electrodeposition: Antagonistic and Synergistic Effects among SPS, Chloride, and Suppressor Additives, J. Phys. Chem. C, 116 (2012) 6913-6924.

[31] N.T.M. Hai, D. Lechner, F. Stricker, J. Furrer, P. Broekmann, Combined Secondary Ion Mass Spectrometry Depth Profiling and Focused Ion Beam Analysis of Cu Films Electrodeposited under Oscillatory Conditions, ChemElectroChem, 2 (2015) 664-671. [32] N.T.M. Hai, P. Broekmann, Smart Hybrid Polymers for Advanced Damascene Electroplating: Combination of Superfill and Leveling Properties, ChemElectroChem, 2 (2015) 1096-1099.

[33] A. Riedo, A. Bieler, M. Neuland, M. Tulej, P. Wurz, Performance evaluation of a miniature laser ablation time-of-flight mass spectrometer designed for in situ investigations in planetary space research, J. Mass Spectrom., 48 (2013) 1-15.

[34] M. Tulej, M. Iakovleva, I. Leya, P. Wurz, A miniature mass analyser for in-situ elemental analysis of planetary material-performance studies, Anal. Bioanal. Chem., 399 (2011) 21852200 .

[35] A. Riedo, M. Neuland, S. Meyer, M. Tulej, P. Wurz, Coupling of LMS with a fs-laser ablation ion source: elemental and isotope composition measurements, J. Anal. At. Spectrom., 28 (2013) 1256-1269.

[36] A. Riedo, S. Meyer, B. Heredia, M.B. Neuland, A. Bieler, M. Tulej, I. Leya, M. Iakovleva, K. Mezger, P. Wurz, Highly accurate isotope composition measurements by a miniature laser ablation mass spectrometer designed for in situ investigations on planetary surfaces, Planet. Space Sci., 87 (2013) 1-13.

[37] M.B. Neuland, S. Meyer, K. Mezger, A. Riedo, M. Tulej, P. Wurz, Probing the Allende meteorite with a miniature laser-ablation mass analyser for space application, Planet. Space Sci., 101 (2014) 196-209.

[38] P. Wurz, D. Abplanalp, M. Tulej, M. Iakovleva, V.A. Fernandes, A. Chumikov, G.G. Managadze, Mass spectrometric analysis in planetary science: Investigation of the surface and the atmosphere, Sol. Sys. Res., 46 (2012) 408-422.

[39] K. Krischer, N. Mazouz, P. Grauel, Fronts, Waves, and Stationary Patterns in

Electrochemical Systems, Angew. Chem. Int. Ed., 40 (2001) 850-869.

[40] P. Strasser, M. Eiswirth, M.T.M. Koper, Mechanistic classification of electrochemical oscillators - an operational experimental strategy, J. Electroanal. Chem., 478 (1999) 50-66. [41] V. Grimaudo, P. Moreno-García, A. Riedo, M.B. Neuland, M. Tulej, P. Broekmann, P. Wurz, High-Resolution Chemical Depth Profiling of Solid Material Using a Miniature Laser Ablation/Ionization Mass Spectrometer, Analytical Chemistry, 87 (2015) 2037-2041. 


\section{Tables}

Table 1 Summary of impurity content (in ppm and at $\%$ ) inside the Imep/SPS-assisted electrodeposited $\mathrm{Cu}$ sample.

\begin{tabular}{lcccccc}
\hline & $\begin{array}{c}C_{\text {Tot }} \\
(\mathrm{ppm})\end{array}$ & $\begin{array}{c}C_{\mathrm{C}, \mathrm{Rel}} \\
(\% \mathrm{at})\end{array}$ & $\begin{array}{c}C_{\mathrm{O}, \mathrm{Rel}} \\
(\% \mathrm{Oat})\end{array}$ & $\begin{array}{c}C_{\mathrm{N}, \mathrm{Rel}} \\
(\% \mathrm{oat})\end{array}$ & $\begin{array}{c}C_{\mathrm{S}, \mathrm{Rel}} \\
(\% \mathrm{oat})\end{array}$ & $\begin{array}{c}C_{\mathrm{Cl}, \mathrm{Rel}} \\
(\% \mathrm{at})\end{array}$ \\
\hline$C(L)$ & 36800 & 47.00 & 33.00 & 10.90 & 7.70 & 1.40 \\
$C(S)$ & 1130 & 27.83 & 51.89 & 12.56 & 5.57 & 2.16 \\
$C(B)$ & 158 & 28.20 & 46.40 & 8.50 & 14.30 & 2.50 \\
\hline
\end{tabular}




\section{Figure Captions}

Figure 1 Reaction scheme demonstrating the SPS surface electrochemistry coupled with the Imep precipitation reaction.

Figure 2 Typical SEM images of the $\mathrm{Cu}$ samples after depth profiling analysis by means of a) SIMS and b) LIMS.

Figure 3 a) Potential versus charge (depth) curve of the galvanostatic copper deposition on a $\mathrm{SiO}_{2}$ supported $\mathrm{Cu}$-wafer coupon at $J=-6 \mathrm{~mA} \mathrm{~cm}^{-2}$. The initial and final stages of the electrodeposition are indicated by arrows. b) Corresponding cross-sectional FIB micrograph of the $\mathrm{Cu}$ film deposited under oscillatory conditions. Extended deltatype grain boundaries are highlighted by white dotted lines and correspond to the sharp potential oscillations in a). c) Negative ion and $\mathrm{MCs}^{+}$mode SIMS depth profiling experiments indicating an oscillatory behavior in the contamination level of $\mathrm{C}, \mathrm{N}, \mathrm{O}$ and $\mathrm{S}$ (in wt\%).

Figure 4 LIMS depth profiles displaying anti-correlated signals of $\mathrm{O}$ and $\mathrm{Cu}$ species.

Figure 5 Representative section of an oxygen LIMS depth profile. Characteristic regimes of the depth profile are denoted as L (sharp delta layers), B (background) and S (satellite peaks).

Figure 6 Graphical representation of the local impurity contents (in ppm) inside the $\mathrm{Cu}$ film as determined by integration of the LIMS intensities. $\mathrm{C}_{\mathrm{L}}$ refers to the sharp delta layers, $C_{S}$ to the satellite peaks and $C_{B}$ to the background in the LIMS depth profiles.

Figure 7 FIB micrograph showing a representative section of the recrystallized $\mathrm{Cu}$ (between two sharp delta layers). The dotted grey line indicates the layer of ablation where the plane of laser irradiation intersects with a relatively low number of grain 
boundaries (denoted B) corresponding to the background contamination level $\left(\mathrm{C}_{\mathrm{B}}\right)$. The dotted red line indicates the layer of ablation with a higher number of grain boundaries (denoted S). The latter corresponds to the appearance of satellite features (S) in the LIMS depth profile in Fig. 5.

Figure 8 (a) Schematics of the two step laser irradiation to address molecular characterization of the $C_{\mathrm{L}}$ impurity layers. (b) Sample mass spectra obtained upon soft laser desorption conditions inside the $C_{\mathrm{L}}$ films in the Imep-Cu(I)-MPS mass window. 


\section{Figure 1}

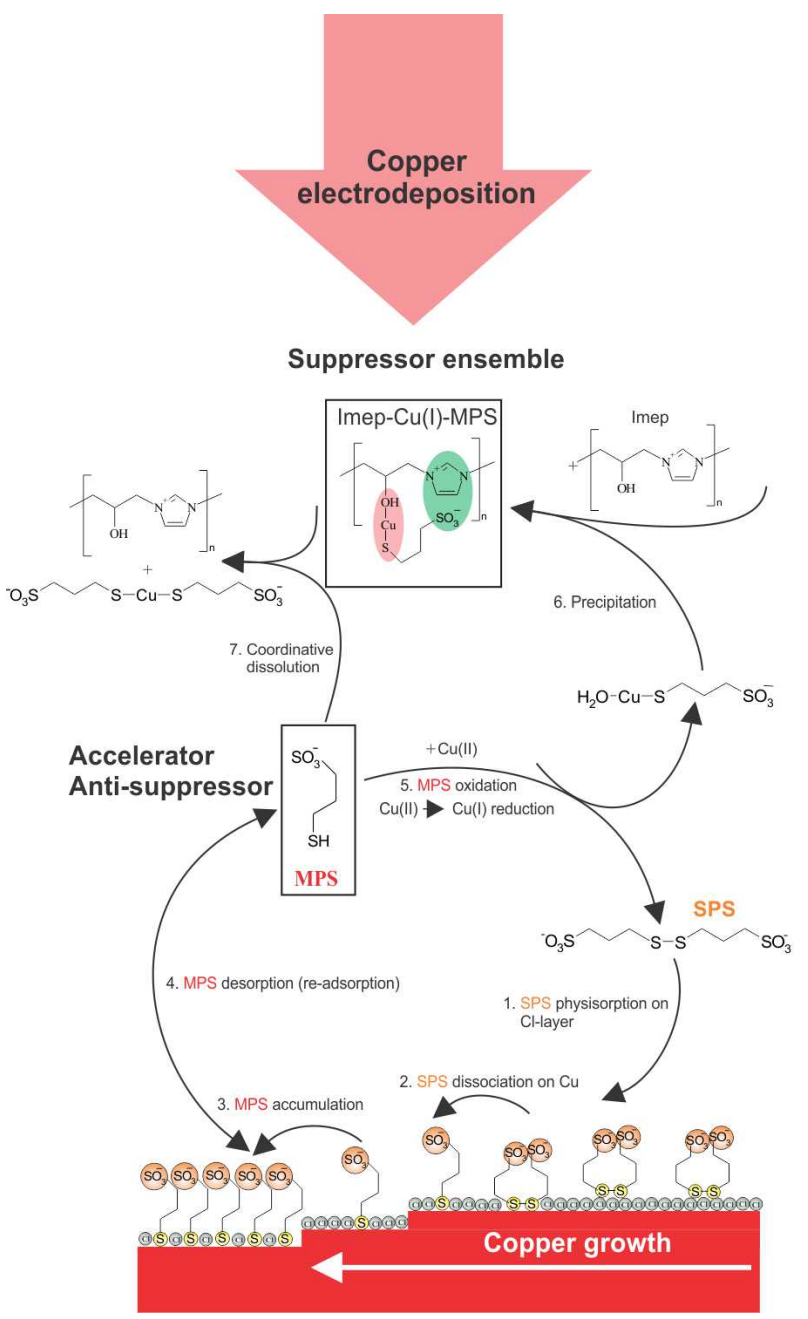

Figure 2 


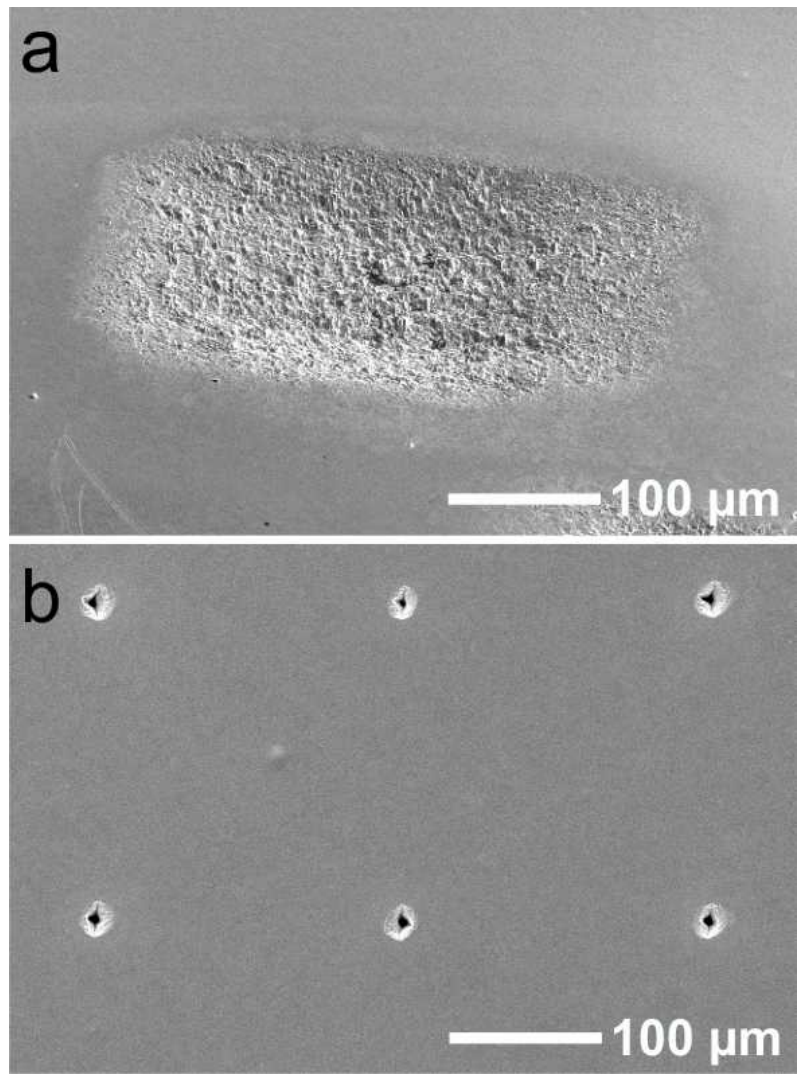

Figure 3 

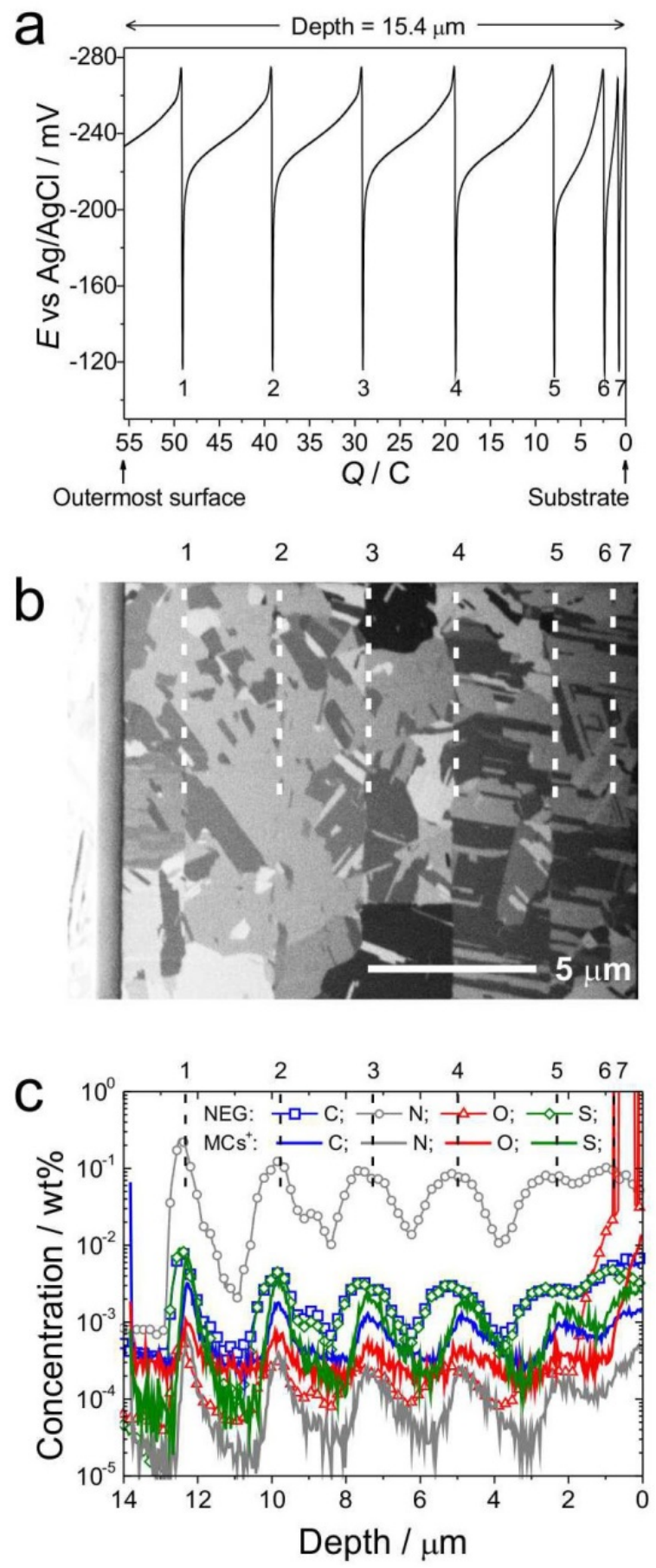

Figure 4 


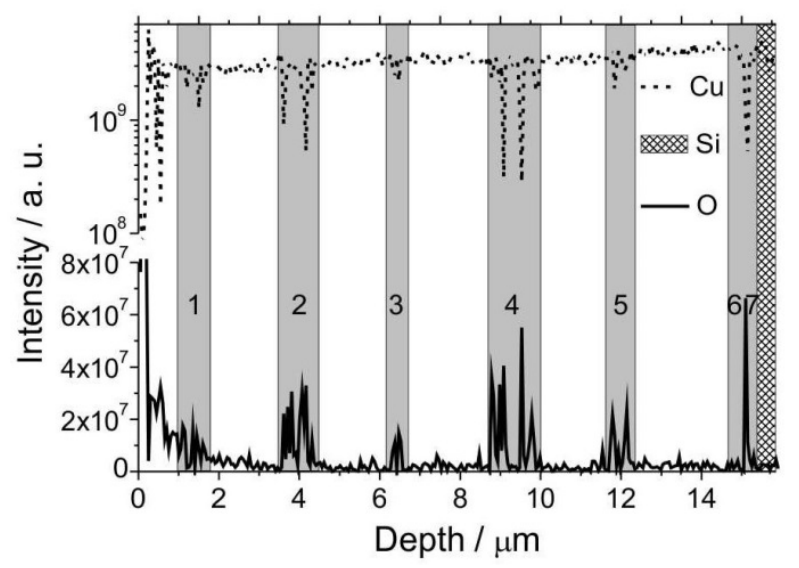

Figure 5 


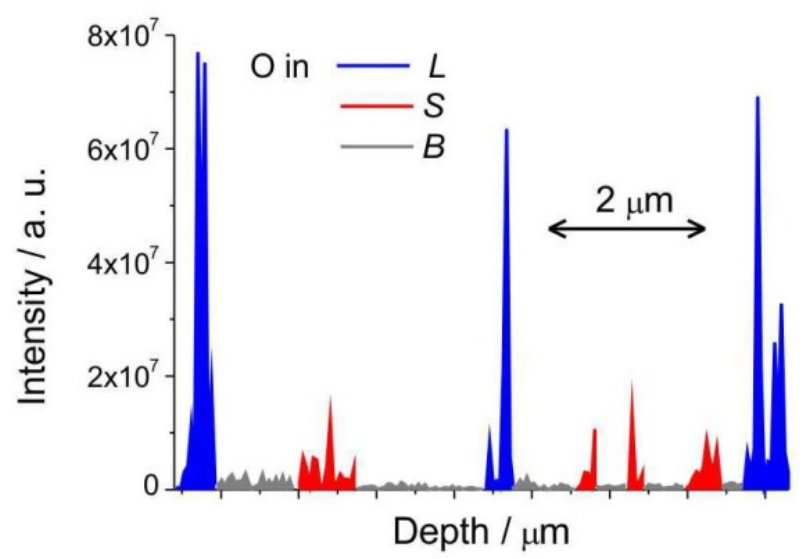

Figure 6 


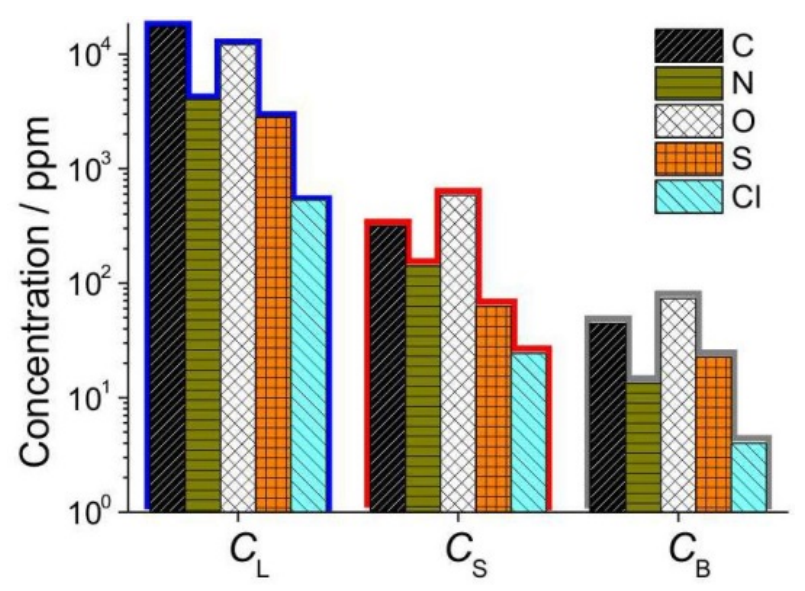

Figure 7 


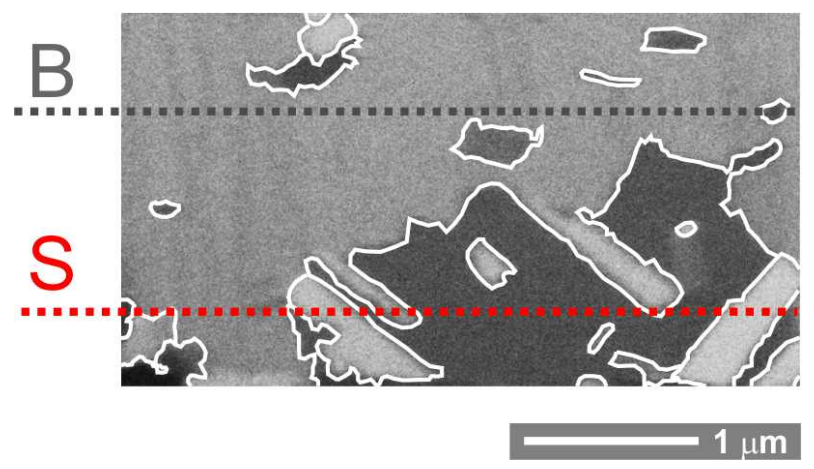

Figure 8 


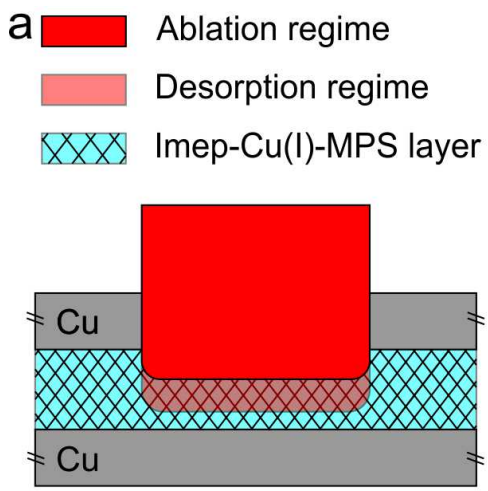

b Complex

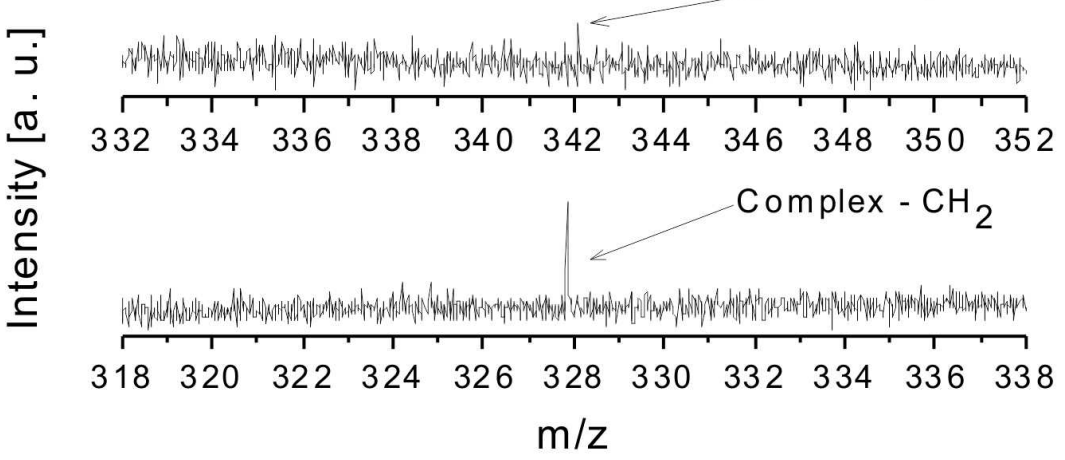

